\title{
Incoherent Synchronization Between Resting State Respiratory Sinus Arrhythmia And Respiratory Movement in Depressed Patients With Suicidal Ideation
}

\author{
Ahsan H Khandoker ${ }^{1}$, Namareq Widatalla ${ }^{1}$, Herbert Jelinek ${ }^{2}$, Kyuichi Niizeki ${ }^{3}$, \\ Leontios Hadjileontiadis ${ }^{1}$ \\ ${ }^{1}$ Khalifa University of Science and Technology, Abu Dhabi, UAE \\ ${ }^{2}$ American Center for Psychiatry and Neurology, Abu Dhabi, UAE \\ ${ }^{3}$ Yamagata University, Yonezawa, Japan
}

\begin{abstract}
The aim of this study is to investigate if the phase synchronization between Respiratory Sinus Arrhythmia (RSA) and respiratory movement in major depressive disorder (MDD) with or without suicidal ideation may be different to a control group during a resting mental state. Diagnoses of MDD were made by the Mini-International Neuropsychiatric Interview (MINI). The subscale (CModule of MINI) with scores more than 9 of 38 were considered with suicidal ideation (SI). The interbeat intervals (RRI) and respiratory movement were extracted from 10 minutes ECG signals of control subjects (10 CONT), $10 \mathrm{MDD}$ subjects with SI (MDDSI+) and 10 without (MDDSI-). Both RRI and respiration were resampled at $10 \mathrm{~Hz}$ and band passed filtered (0.10-0.4 $\mathrm{Hz}$ ). Hilbert transform was applied to extract instantaneous phases of the RSA and respiration. Then time dependent phase coherence $(\lambda)$ between RSA and Respiration was obtained. HRV indices were also calculated. Higher breathing frequency and lower amplitude of RSA (ARSA), lower $\lambda$ values and HF power were found in the MDDSI+ group compared to the MDDSI- group $(p<0.05)$ and CONT group $(p<0.01)$. In this preliminary study we conclude that the resting mental state in MDD patients exerts an influence on RSA oscillations with respect to respiratory movements.
\end{abstract}

\section{Introduction}

Over $90 \%$ of people who die by suicide have clinical depression or another diagnosable mental disorder $[1,2]$. Individuals with major depressive disorder (MDD) with symptoms of suicidal risk might have been undiagnosed because of persistent ignorance about depression. Misperceptions by the public, and even some health providers, who interpret the disease to be a personal weakness in character, often lead to painful stigmatization of sufferers, and treatment avoidance. In the United Arab Emirates, apathy towards seeking professional mental health services by UAE citizens is associated with societal stigma, lack of awareness of mental health and lack of confidence in mental health-care providers [3]. Therefore, it has been recommended that physiological evidence-based diagnostic methods and interventions need to be implemented at a population, sub-population and individual level to prevent suicide and suicide attempts. Suggestions have been made that alternative diagnostic tools be used in conjunction with a psychiatric evaluation, or as a stand-alone screening, as part of a community health initiative; which may provide an additional source of information, allowing individuals at risk to be assessed.

Heart rate variability (HRV) has been used as an indicator of autonomic nervous system function. In particular, respiratory sinus arrhythmia (RSA), the highfrequency component of HRV, has been linked to cardiac vagal tone in studies using autonomic blockade [4]. Thus RSA has been recognized as an index of the vagal-cardiac nerve traffic [5]. Decreased HRV [6] and RSA [7] have been associated with MDD subjects, which are signs of a low level of parasympathetic modulations. The magnitude of the RSA varies among individual depending on age, gender, and physical fitness [8].

Recently cardiorespiratory phase synchronization has recently received attention $[9,10]$. The analysis of phase synchronization between RSA and breathing may provide a new index for evaluating the psychological State of patients with MDD. It was reported that mental challenge may alter phase synchronization among hemodynamic 
variables and respiration [11], yet quantitative analysis for the phase relationship between RSA and respiration in depressed patients has not been determined in conjunction with the autonomic indexes. In this study, we hypothesized that altered psychophysiology in patients with MDD may distort the phase relationship between respiration and RSA, and if this is the case, phase synchronization between respiration and RSA could provide a useful and convenient measure for evaluating depression severity and suicidal risk.

\section{Methods}

\subsection{Subjects and psychiatric assessment}

Ten (10) non-depressed subjects and twenty (20) patients with a primary diagnosis of MDD were included in this study. Participants were recruited from the outpatient clinic at the American Center for Psychiatry and Neurology in Abu Dhabi. The study was approved by Al Ain District Ethics Committee, and all participants provided written informed consent. Diagnoses, including history of suicidal ideation (SI), were made by a consultant Psychiatrist (VL) using the Mini-International Neuropsychiatric Interview (MINI) [12] and the severity of clinical depression was assessed using the structured interview guide for the Hamilton Depression Rating Scale (HAM-D) [13]. All patients recruited in this study were diagnosed as MDDSI+ (MDD subjects with suicidal ideation) and MDDSI- (MDD subjects without suicidal ideation) at their first visit to the psychiatric clinic. The suicidality module of MINI, also known as the ' $\mathrm{C}$ module' was used in this study to estimate the suicidal ideation. This module is made up of nine questions. Questions C1 to C8 were used to assess events in the past month, while question C9 was used to assess for suicide attempt in a patient's lifetime. The module assessed suicidal behavior including intent, planning and attempting suicide. The minimum score for the $\mathrm{C}$ module is zero (0) and the maximum score possible is 38 . In this study, MDD patients with scores of more than 9 were considered to be MDDSI+ patients.

ECG and piezoelectric belt based thoracic movement signals were recorded from all subjects for 10 minutes (Powerlab, AD Instruments, Australia) at sampling rate of $1000 \mathrm{~Hz}$.

\subsection{Data analyses}

Both RRI and respiration were resampled at $10 \mathrm{~Hz}$ and band passed filtered $(0.10-0.4 \mathrm{~Hz})$. Hilbert transform was applied to extract instantaneous phases of the RSA [ $\left.\Phi_{R S A}\left(t_{k}\right)\right]$ and respiration $\left[\Phi_{R E S P}\left(t_{k}\right)\right]$. Then time dependent phase coherence $(\lambda)$ between RSA and Respiration was obtained by the following equation.

$$
\lambda\left(t_{k}\right)=\left|\frac{1}{N} \sum_{j=k-\frac{N}{2}}^{k+\frac{N}{2}} e^{\left[\Phi_{R S A}\left(t_{k}\right)-\Phi_{R E S P}\left(t_{k}\right)\right] \bmod 2 \pi}\right|^{2}
$$

$\mathrm{N}$ denotes number of data samples. The $\lambda$ value ranges from 0 (lowest) 1(highest) coupling.

\subsection{HRV}

Spectral analysis was performed on linearly resampled (4 Hz) time series using Welch's method [14]. The 256point fast Fourier transform was repeatedly computed with $50 \%$ overlap between adjacent segments. Then the spectral power of each segment was computed and averaged. Hanning window was applied to avoid spectral leakage. Subsequently, spectral powers in the low frequency (LF) band $(0.04-0.15 \mathrm{~Hz})$ and high frequency (HF) band $(0.15-0.40 \mathrm{~Hz})$ were obtained by integration [15].

\subsection{Statistics}

In this study the nonparametric Mann-Whitney U-test was performed to check the differences between healthy (CONT) vs. MDD patients with suicidal ideation (MDDSI+), (CONT) vs. MDD patients without suicidal ideation (MDDSI-) and MDD patients with suicidal ideation (MDDSI+) vs. MDD patients without suicidal ideation (MDDSI-). Values of $\mathrm{p}<0.05$ were considered as significant.

\section{Results and Discussion}

Figure 1 shows three examples of R-R intervals (RRI), Respiratory movement (Resp), Instantaneous phases for breathing and RRI $(\phi)$ and phase coupling coefficient $(\lambda)$ from three groups.

Table 1 summarizes the analysis results. Higher Breathing frequency (fR) [fR (breaths/min): $20 \pm 4$ (MDDSI+), $17 \pm 2$ (MDDSI-), 16 $\pm 3(\mathrm{CONT})]$, and lower amplitude of RSA(ARSA) [ARSA (ms): $18 \pm 3$ (MDDSI+), $20 \pm 1$ (MDDSI-), 24 $\pm 3(\mathrm{CONT})]$, lower $\lambda$ values $\quad[0.51 \pm 0.5 \quad(\mathrm{MDDSI}+), \quad 0.54 \pm 0.4$ (MDDSI-), $0.71 \pm 0.2(\mathrm{CONT})]$ and lower HF power of RRI [ms2: 402 $\pm 101 \quad$ (MDDSI+), $569 \pm 203 \quad$ (MDDSI-), 
$1050 \pm 490(\mathrm{CONT})]$ were found in MDDSI+ group as compared to MDDSI- group $(\mathrm{p}<0.05)$ and CONT group $(\mathrm{p}<0.01)$. The $\lambda$ was significantly $(\mathrm{p}<0.05)$ positively correlated with ARSA $(\mathrm{r}=0.65)$ and the HF power $(\mathrm{r}=0.55)$.
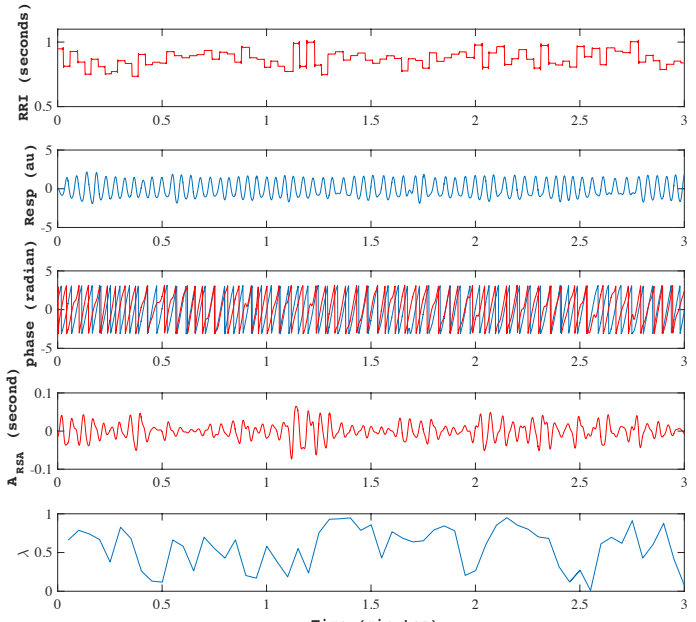

(A)
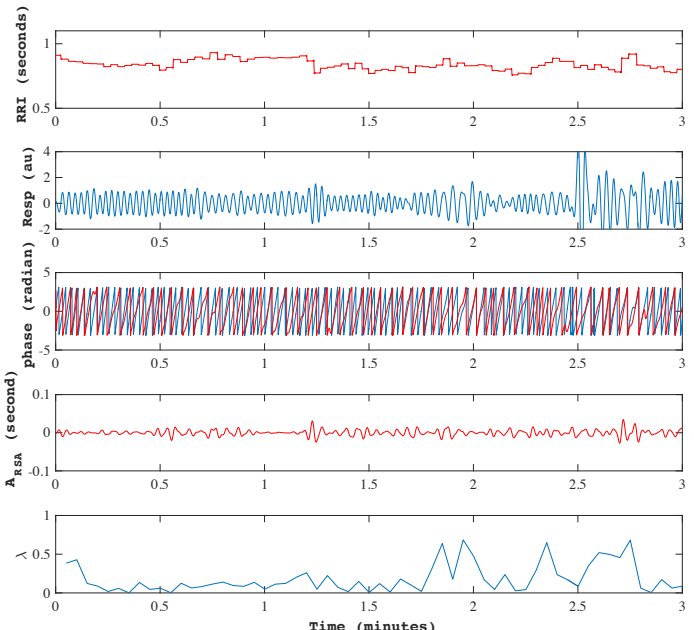

(B)
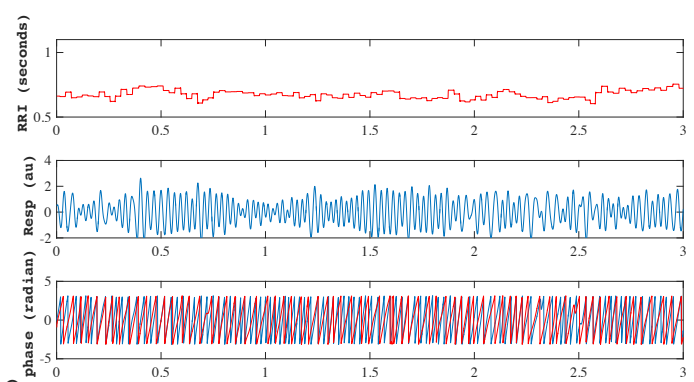

ซึ: 0.1
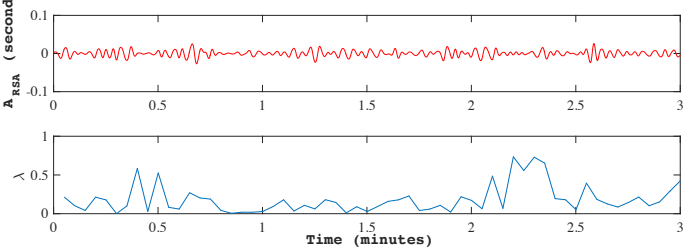

(C)

Fig.1. R-R intervals (RRI), Respiratory movement (Resp), Instantaneous phases for breathing and RRI $(\phi)$ and phase coupling coefficient $(\boldsymbol{\lambda})$ from examples of control group (A), MDDSI-group (B) and MDDSI+ group (C).

Table 1: Mean \pm SD of cardiorespiratory and HRV variables. * means significant difference $(\mathrm{p}<0.05)$ between Control/MDDSI, ** MDDSI-/SI+, *** MDDSI+/Control.

\begin{tabular}{|c|c|c|c|}
\hline & $\begin{array}{l}\text { Control } \\
(10)\end{array}$ & $\begin{array}{l}\text { MDDSI- } \\
(10)\end{array}$ & $\begin{array}{l}\text { MDDSI } \\
+(10) \\
\end{array}$ \\
\hline RRI (ms) & $\begin{array}{c}850 \pm 90 \\
(* * *)\end{array}$ & $830 \pm 64$ & $770 \pm 87$ \\
\hline $\begin{array}{l}\quad \mathrm{f}_{\mathrm{R}} \\
\text { Breathing } \\
\text { frequency } \\
\text { (breaths } / \mathrm{min} \text { ) }\end{array}$ & $\begin{array}{l}16 \pm 3 \\
(* * *)\end{array}$ & $17 \pm 2$ & $20 \pm 4$ \\
\hline $\mathrm{A}_{\mathrm{RSA}}(\mathrm{ms})$ & $\begin{array}{l}24 \pm 3 \\
(* * *)\end{array}$ & $\begin{array}{c}20 \pm 3 \\
(* *)\end{array}$ & $18 \pm 2$ \\
\hline$\lambda$ & $\begin{array}{c}0.71 \pm 0.2 \\
(* * *)\end{array}$ & $\begin{array}{c}0.54 \pm 0.4 \\
(* *)\end{array}$ & $0.51 \pm 0.5$ \\
\hline $\mathrm{LF}\left(\mathrm{ms}^{2}\right)$ & $\begin{array}{c}990 \pm 590 \\
(* * *)\end{array}$ & $670 \pm 390$ & $490 \pm 590$ \\
\hline $\mathrm{HF}\left(\mathrm{ms}^{2}\right)$ & $\begin{array}{c}1050 \pm 490 \\
(* * *)\end{array}$ & $569 \pm 203$ & $402 \pm 101$ \\
\hline
\end{tabular}

The present study demonstrated that the interaction of respiration and heart rate with phase-lag variations between RSA and respiration in MDD patients with SI as measured by $\lambda$ is the lowest, in addition to an attenuation of RSA. These changes are accompanied by increases in 
$\mathrm{f}_{\mathrm{R}}$ in the MDDSI+ group as compared to control one. The $\lambda$ showed significant positive correlation with autonomic vagal activity indexes of $A_{R S A}$ and the $H F$ component of HRV, suggesting a possibility that $\lambda$ may provide information about the parasympathetic dysfunction in depression.

In this preliminary study we conclude that resting mental state in patients with MDD could exert an influence on RSA oscillations by decreasing the amplitudes, inducing incoherent phase lag with respect to respiratory movements. These findings could help explore clinico-biological patterns in MDD and assess the efficacy of various treatment modalities for a variety of mental disorders by reverting the phase synchronization index between RSA and Respiratory movement back to normal level.

\section{Acknowledgements}

This study was supported by Al Jalila Foundation awarded to Leontios Hadjileontiadis.

\section{References}

[1] Leenaars, A. A. (1996). Suicide: A multidimensional malaise. Suicide and Life-Threatening Behavior, 26(3), 221-236.

[2] Soloff, P. H., Lynch, K. G., Kelly, T. M., Malone, K. M., \& Mann, J. J. (2000). Characteristics of suicide attempts of patients with major depressive episode and borderline personality disorder: a comparative study. American Journal of Psychiatry, 157(4), 601-608.

[3] Chowdhury, N. (2016). Integration between mental health-care providers and traditional spiritual healers: contextualising Islam in the twenty-first century. Journal of Religion and Health, 1-7.

[4] Akselrod S, Gordon D, Ubel FA, Shannon DC, Berger AC, Cohen CJ Power spectrum analysis of heart rate fluctuation: a quantitative probe of beat-to-beat cardiovascular control. Science 213: 220-222, 1981.

[5] Grossman P, Wilhelm FH, Spoerle M. Respiratory sinus arrhythmia, cardiac vagal control, and daily activity. Am J Physiol Heart Circ Physiol 287: H728-H734, 2004.

[6] Khandoker, A. H., Luthra, V., Abouallaban, Y., Saha, S., Ahmed, K. I., Mostafa, R., ... \& Jelinek, H. F. (2017). Predicting depressed patients with suicidal ideation from ECG recordings. Medical \& biological Engineering \& Computing, 55(5), 793-805.

[7] Bernardi L, Wdowczyk-Szulc J, Valenti C, Castoldi S, Passino C,Spadacini G, Sleight P. Effects of controlled breathing, mental activity and mental stress with or without verbalization on heart rate variability. J Am Coll Cardiol 35: 1462-1469, 2000.

[8] Lamine SB, Calabrese P, Perrault H, Dinh TP,
Eberhard A, Benchtrit G. Individual differences in respiratory sinus arrhythmia. Am J Physiol Heart Circ Physiol 286: H2305-H2313, 2004.

[9] Schäfer C, Rosenblum MG, Kurths J, Abel HH. Heartbeat synchronized with ventilation. Nature 392: 239-249, 1998.

[10] Schäfer C, Rosenblum MG, Abel HH, Kurths J. Synchronization in the human cardiorespiratory system. Phys Rev E Stat Phys Plasmas Fluids Relat Interdiscip Topics 60: 857-870, 1999.

[11] Lackner HK, Papousek I, Batzel JJ, Roessler A, Scharfetter H, Hinghofer-Szalkay H. Phase synchronization of hemodynamic variables and respiration during mental challenge. Int J Psychophysiol 79: 401-409, 2011.

[12] Sheehan, D. V., Lecrubier, Y., Sheehan, K. H., Amorim, P., Janavs, J., Weiller, E., ... \& Dunbar, G. C. (1998). The Mini-International Neuropsychiatric Interview (MINI): the development and validation of a structured diagnostic psychiatric interview for DSM-IV and ICD-10. Journal of Clinical Psychiatry, 59, 22-33

[13] Williams, J. B. (1988). A structured interview guide for the Hamilton Depression Rating Scale. Archives of General Psychiatry, 45(8), 742-747.

[14] Welch, P. D. (1967). The use of fast Fourier transform for the estimation of power spectra: A method based on time averaging over short, modified periodograms. IEEE Transactions on Audio and Electroacoustics, 15(2), 70-73.

[15] Camm, A. J., Malik, M., Bigger, J. T., Günter, B., Cerutti, S., \& Choen, R. (1996). Task force of the European Society of Cardiology and the North American Society of Pacing and Electrophysiology. Heart rate variability: standards of measurement, physiological interpretation and clinical use. Circulation, 93(5), 10431065 .

Address for correspondence.

Name. Dr Ahsan Khandoker

Full postal address. Department of Biomedical Engineering, Khalifa University, Abu Dhabi, PO Box 127788, UAE.

E-mail address (optional). ahsan.khandoker@kustar.ac.ae 